\title{
Gender disparities among medical students choosing to pursue careers in medical research: a secondary cross-sectional cohort analysis
}

\author{
Austin Snyder ${ }^{1}$, David Xiang ${ }^{1}$, Alison Smith², Shannon Esswein ${ }^{3}$, Omar Toubat ${ }^{2}$, John Di Capua ${ }^{4}$, \\ Jennifer M. Kwan ${ }^{5^{*}}$ and Dania Daye ${ }^{4^{*}}$
}

\begin{abstract}
Background: Though the proportion of women in medical schools has increased, gender disparities among those who pursue research careers still exists. In this study, we seek to better understand the main factors contributing to the existing gender disparities among medical students choosing to pursue careers in medical research.

Methods: A secondary cross-sectional cohort analysis of previously published data was conducted using a 70-item survey that was sent to 16,418 medical students at 32 academic medical centers, and was IRB exempt from the need for ethical approval at the University of Illinois at Chicago and the University of Pennsylvania. Data was collected from September 2012 to December 2014. Survey results were analyzed using chi-square tests and Cramer's V to determine gender differences in demographic characteristics (training stage, race/ethnicity, marital status, parental status, financial support, and parental career background), career sector choice, career content choice, specialty choice, foreseeable career obstacles, and perceptions about medical research careers.

Results: Female respondents were more likely to be enrolled in MD-only programs, while male respondents were more likely to be enrolled in MD/PhD programs. More male students selected academia as their first-choice career sector, while more female respondents selected hospitalist as their first-choice career sector. More female respondents identified patient care and opportunities for community service as their top career selection factors, while more male respondents identified research and teaching as their top career selection factors. Student loan burden, future compensation, and work/life balance were the most reported obstacles to pursuing a career in medical research.

Conclusions: There are many factors from a medical student's perspective that may contribute to the existing gender disparities in pursuing a career in medical research. While much progress has been made in attracting nearly equal numbers of men and women to the field of medicine, active efforts to bridge the gap between men and women in medical research careers are needed.
\end{abstract}

Keywords: Gender disparities, Research, Academic medicine, Work-life balance

*Correspondence: kwanjen@gmail.com; ddaye@mgh.harvard.edu

${ }^{4}$ Department of Interventional Radiology, Massachusetts General

Hospital, 55 Fruit Street, Boston, MA 02114, USA

${ }^{5}$ Section of Cardiovascular Medicine, Yale School of Medicine, 333 Cedar

Street, New Haven, CT 06510, USA

Full list of author information is available at the end of the article

\section{Introduction}

Physician-scientists have long been considered an endangered species and the female physician-scientist an even more rare entity [1]. Despite efforts to attract physicians to medical research, interest has continued to dwindle, especially among female students. Furthermore, women also continue to be underrepresented in leadership and 
administrative roles in academic medicine [2]. While the causes of these phenomena have long been debated and are certainly multi-factorial, no solutions have been realized.

Furthermore, for reasons that are not well understood, the interest in medical research has been dwindling [35]. According to a 2014 report from the NIH, only $1.5 \%$ of MDs consider research their primary focus with even fewer physicians receiving funding as principal investigators on $\mathrm{NIH}$ grants $(0.9 \%)$, split evenly between MDs and MD-PhDs [6]. Whereas the number of nonphysician $(\mathrm{PhD}) \mathrm{NIH}$-funded investigators has increased by $50 \%$ over the last 20 years, the number of NIH-funded physician-scientists has essentially remained constant.

The stagnating physician-scientist workforce has also failed to keep pace with the increase in racial and gender diversity of its MD counterpart. The ever-growing body of evidence continues to support the importance of achieving greater diversity in the biomedical workforce [7]. However, the pipeline remains leaky with striking losses of female talent at higher levels of academic medicine $[8,9]$. Historically, the female physician was a rarity. In the 1970s, the proportion of women graduating from US medical schools nearly tripled by the end of that decade [10]. Today, women comprise $46 \%$ of residents, yet the proportion of women at the rank of full professor (12\%) remains far below that of men [11].

Unfortunately, women are much less likely than their male counterparts to express interest in a career in medical research altogether, at either matriculation or graduation. Furthermore, women who initially express interest in pursuing research as part of their careers are more likely to lose their research career aspirations throughout medical school [12]. The reasons for these disparities are certainly multifactorial and likely include factors such as lack of adequate role models, gender discrimination/bias, and work-life balance, but there remain many inconsistencies in the contribution of these factors to this alarming trend [13]. Although previous studies have identified factors influencing interest in research careers among $\mathrm{MD}$ and $\mathrm{MD} / \mathrm{PhD}$ students [14], this study aims to provide a secondary analysis of this data to investigate gender differences among various factors contributing to medical students' interest in pursuing medical research.

\section{Methods}

\section{Data collection}

The study was reviewed and IRB exempt from the need for ethical approval at the University of Illinois at Chicago and the University of Pennsylvania. All methods were carried out in accordance with relevant guidelines and regulations. A secondary cross-sectional cohort analysis of previously published data was conducted using a 70-item survey that was designed with feedback from a survey design team at the University of Illinois at Chicago $[14,15]$. Data were collected using an online survey tool (SurveyMonkey, www.surveymonkey.com) (Appendix I). The survey tool was piloted at 5 institutions for validation of the survey which lasted 18 months (2011 to 2012) [14]. This data was not included in the present study.

Data collection for the present study was collected from 2012 to 2014. The survey was sent in September 2012 via e-mail to $16,418 \mathrm{MD}$ and $\mathrm{MD} / \mathrm{PhD}$ students at $32 \mathrm{US}$ academic medical centers through student listservs and the institutional representatives of the American Physician Scientists Association (APSA). There was a phased deployment of the survey during this time with the addition of different institutions each year. Three reminders were sent per institution during the study period.

All identifying information was anonymized by the survey collection tool. Furthermore, the survey collection tool did not allow for more than 1 response from each IP address. To participate in the study, respondents had to be students enrolled in a medical school or graduate school program, or taking a year off for research, which was reflected in the responses to the survey. The denominator of total responses used to calculate the response rate represents the cumulative number of students that the survey was sent out to at the participating institutions. Participants had the option of entering their institutional email address for a chance to receive a $\$ 50$ Amazon gift certificate. E-mail addresses were kept separate from survey responses to maintain the anonymity of responses.

$\mathrm{MD} / \mathrm{PhD}$ students were identified through how they paid for medical school as being sponsored by an $\mathrm{MD} /$ $\mathrm{PhD}$ program. MD candidates interested in researchintensive careers (MD-RI) were identified by their career intentions of wanting a research to clinical duty ratio of $50 \%$ or greater, which reflects the NIH guidelines for surgeon scientists. Medical students (years 1-4) were defined as those enrolled in a US medical school. Graduate school students (years 1-5+) were defined as those enrolled in an MD/PhD program. Students taking a year out for research, or a one-year graduate school program were separately categorized by the survey. The primary hypothesis was that there is a difference in barriers to pursuing careers in medical research based on gender. The primary outcome was the various factors contributing to the gender disparities in medical research careers.

\section{Statistical analysis}

Survey results were analyzed to determine significant gender differences in demographic characteristics (training stage, race/ethnicity, marital status, parental status, financial support, and parental career background), 
career sector choice, career content choice, specialty choice, foreseeable career obstacles, and perceptions about medical research careers. Chi-squared tests were used to measure the significance of associations between categorical variables. Where data did not meet minimum expected cell counts, Fisher's exact test was performed. Cramer's V analysis was used to estimate the effect size of statistically significant tests between male and female respondents. The strength of association for Cramer's $\mathrm{V}$ was categorized as follows: $\leq 0.05$ (very weak), $>0.05$ (weak), $>0.10$ (moderate), $>0.15$ (strong), and $>0.25$ (very strong) [16]. All tests were performed using SPSS. All tests of significance were 2 -sided and $p<0.05$ was considered significant. Acute care specialties included pulmonary critical care, anesthesiology, and emergency medicine.

\section{Results}

\section{Demographics}

There were 4433 respondents (27\% response rate). Demographic characteristics (gender, training stage, race/ethnicity, marital status, parental status, financial support, and parental career background) of respondents are summarized in Table 1.

\section{Gender}

Among all respondents, there were more females (2328, $56.3 \%)$ than males $(1795,43.4 \%)$. Female respondents were more likely to be enrolled MD-only programs (1568, $67.4 \%$ versus $1052,58.6 \%$ ) while male respondents were more likely to be enrolled in $\mathrm{MD} / \mathrm{PhD}$ programs (459, $25.6 \%$ versus $394,16.9 \%)$. In contrast, an equal proportion of female $(366,15.7 \%)$ and male respondents $(284$, $15.8 \%)$ self-identified to be MD-RI as defined by intending a $>50 \%$ research/clinical ratio. $P$-value $<0.001$ unless otherwise stated (Table 1).

\section{Training stage}

Survey responses came from students in all stages of MD and $\mathrm{MD} / \mathrm{PhD}$ programs, including all medical school years, five different graduate school years, and students in a research year program. No significant difference in distribution between males and females within each specific stage of training was observed $(p=0.20)$ (Table 1$)$.

\section{Race/ethnicity}

The majority of respondents were white (2850, 71.3\%). Among male students, significantly more white (1263, $72.8 \%$ versus $1587,70.0 \%)$ and multiracial students $(258,14.9 \%$ versus $303,13.4 \%)$ responded compared to females. In contrast, among female respondents, more identified as black (114, 5.0\% versus 52, 3.0\%) or Asian
(259, 11.4\% versus $159,9.2 \%)$ compared to their male counterparts $(p=0.002)$ (Table 1$)$.

\section{Marital status}

Most survey respondents were not married/partnered (2959, 73.8\%) versus married/partnered students (1050, $26.2 \%)$. There were no gender differences between partnered and not partnered students $(p=0.07)$ (Table 1$)$.

\section{Parental status}

A majority of respondents did not have children (3779, 94.3\%) compared to those who had children (229, 5.7\%). 132 (7.6\%) of male respondents reported having children compared to $97(4.3 \%)$ of female respondents $(p<0.0001)$ (Table 1).

\section{Financial support}

More males than females paid for their medical training exclusively through program (i.e. $\mathrm{MD} / \mathrm{PhD}$ or $\mathrm{DO} / \mathrm{PhD}$ ) sponsorships (403, $22.9 \%$ versus $345,15.1 \%$ ), scholarships (171, 9.7\% versus $210,9.2 \%)$, grants $(36,2.1 \%$ versus 37 , $1.6 \%$ ), national services (31, $1.8 \%$ versus $19,0.8 \%$ ), and work $(4,0.2 \%$ versus $2,0.1 \%)$. In contrast, more female than male respondents depended upon loans (1238, $54.3 \%$ versus $874,49.7 \%$ ), personal savings ( $27,1.2 \%$ versus $18,1.0 \%)$, and family/partner support $(398,17.5 \%$ versus $223,12.7 \%)(p<0.0001)$ (Table 1$)$.

\section{Career intentions \\ Career sector}

More male students selected academia (833, 49.7\% versus $1008,46.7 \%$ ) as their first-choice career compared to females. In contrast, more female respondents chose hospitalist $(432,20.0 \%$ versus $254,15.2 \%)$ careers as their top selection relative to males $(p=0.0004)$ (Fig. 1a).

\section{Career content}

More females desired clinical duties $(1526,70.1 \%$ versus $1013,61.1 \%)$ and advocacy work $(73,3.4 \%$ versus 16 , $1.0 \%)$ as their first career intention compared to male students. Male students, in contrast, chose translational research (242, 14.6\% versus 200, 9.2\%), basic research (130, $7.8 \%$ versus $75,3.4 \%$ ), and therapeutics/diagnostics work $(44,2.7 \%$ versus $27,1.2 \%)$ as their top career intention compared to females $(p<0.0001)$ (Fig. 1b).

\section{Residency specialties: 1st specialty of interest}

Significantly more male students preferred surgical specialties $(471,28.3 \%$ versus $499,23.3 \%)$, emergency medicine $(168,10.1 \%$ versus $153,7.1 \%)$, and radiology (90, $5.4 \%$ versus $75,3.5 \%$ ) relative to females, while more female respondents chose medical specialties (1245, 
Table 1 Demographics of Female and Male Respondents

\begin{tabular}{|c|c|c|c|c|}
\hline Demographic & Female, n (\%) & Male, $\mathrm{n}(\%)$ & $P$-value ${ }^{\mathrm{b}}$ & Cramer's V \\
\hline Gender Distribution & $2328(56.3 \%)$ & $1795(43.4 \%)$ & & \\
\hline Training program & & & $<0.001$ & 0.11 \\
\hline MD-RI & $366(15.7 \%)$ & $284(15.8 \%)$ & & \\
\hline $\mathrm{MD} / \mathrm{PhD}$ & $394(16.9 \%)$ & $459(25.6 \%)$ & & \\
\hline MD Only & $1568(67.4 \%)$ & $1052(58.6 \%)$ & & \\
\hline TOTAL & $2328(100 \%)$ & 1795 (100\%) & & \\
\hline Training stage $^{a}$ & & & 0.20 & N/A \\
\hline Medical School Year 1 & $657(28.4 \%)$ & $502(28.2 \%)$ & & \\
\hline Medical School Year 2 & $576(24.9 \%)$ & $462(26.0 \%)$ & & \\
\hline Medical School Year 3 & $392(17.0 \%)$ & $281(15.8 \%)$ & & \\
\hline Medical School Year 4 & $407(17.6 \%)$ & $271(15.2 \%)$ & & \\
\hline Graduate School Year & $5(0.2 \%)$ & $5(0.3 \%)$ & & \\
\hline Year Out for Research & $61(2.6 \%)$ & $39(2.2 \%)$ & & \\
\hline Graduate School Year 1 & $64(2.8 \%)$ & $69(3.9 \%)$ & & \\
\hline Graduate School Year 2 & $49(2.1 \%)$ & $54(3.0 \%)$ & & \\
\hline Graduate School Year 3 & $44(1.9 \%)$ & $40(2.3 \%)$ & & \\
\hline Graduate School Year 4 & $46(2.0 \%)$ & $42(2.4 \%)$ & & \\
\hline Graduate School Year 5 or more & $11(0.5 \%)$ & $14(0.8 \%)$ & & \\
\hline TOTAL & $2312(100 \%)$ & 1779 (100\%) & & \\
\hline Race & & & 0.002 & 0.07 \\
\hline White & 1587 (69.9\%) & $1263(72.8 \%)$ & & \\
\hline Black or African American & $114(5.0 \%)$ & $52(3.0 \%)$ & & \\
\hline American Indian or Alaska Native & $6(0.3 \%)$ & $4(0.2 \%)$ & & \\
\hline Asian or Pacific Islander & $259(11.4 \%)$ & $159(9.2 \%)$ & & \\
\hline Multi-racial or Other & $303(13.4 \%)$ & $258(14.9 \%)$ & & \\
\hline TOTAL & $2269(100 \%)$ & $1736(100 \%)$ & & \\
\hline Marital status & & & 0.07 & N/A \\
\hline Married/Partnered & $569(25.1 \%)$ & $481(27.6 \%)$ & & \\
\hline Not Married/Partnered & $1698(74.9 \%)$ & $1261(72.4 \%)$ & & \\
\hline TOTAL & $2267(100 \%)$ & $1742(100 \%)$ & & \\
\hline Parental status & & & $<0.0001$ & 0.07 \\
\hline Has a child/children (of 4041) & $97(4.3 \%)$ & $132(7.6 \%)$ & & \\
\hline Does NOT have a child/children & $2168(95.7 \%)$ & $1611(92.4 \%)$ & & \\
\hline TOTAL & $2265(100 \%)$ & $1743(100 \%)$ & & \\
\hline Primary source of medical school funding & & & $<0.0001$ & 0.13 \\
\hline MD-PhD or DO-PhD sponsored only & $345(15.1 \%)$ & $403(22.9 \%)$ & & \\
\hline Scholarships & $210(9.2 \%)$ & $171(9.7 \%)$ & & \\
\hline Grants & $37(1.6 \%)$ & $36(2.1 \%)$ & & \\
\hline Loans & $1238(54.3 \%)$ & $874(49.7 \%)$ & & \\
\hline National Service & $19(0.8 \%)$ & $31(1.8 \%)$ & & \\
\hline Personal Savings & $27(1.2 \%)$ & $18(1.0 \%)$ & & \\
\hline Family/Partner Support & $398(17.5 \%)$ & $223(12.7 \%)$ & & \\
\hline Work & $2(0.1 \%)$ & $4(0.2 \%)$ & & \\
\hline Other & $3(0.1 \%)$ & $0(0.0 \%)$ & & \\
\hline TOTAL & $2279(100 \%)$ & $1760(100 \%)$ & & \\
\hline
\end{tabular}

a Excluding Other/NA

b Male versus female responses were compared using chi-squared tests and Fisher's exact tests where appropriate

c Cramer's V was used to measure effect size between male and female respondents 
$58.0 \%$ versus $782,47.0 \%)$ as their top intended specialty $(p<0.0001)$ (Fig. 1c).

\section{Career selection factors}

More male respondents identified research $(255,15.4 \%$ versus $169,7.7 \%)$, teaching $(60,3.6 \%$ versus $41,1.9 \%)$, financial security $(110,6.6 \%$ versus $52,2.4 \%)$ and autonomy $(61,3.7 \%$ versus $33,1.5 \%)$ as the top career selection factors. In comparison, more female respondents identified patient care $(809,37.1 \%$ versus $510,30.8 \%)$, community service $(93,4.3 \%$ versus $29,1.8 \%$ ) and work life balance $(855,39.2 \%$ versus $539,32.6 \%)$ as the top career selection factors $(p<0.0001)$ (Table 2$)$.

\section{Obstacles}

Foreseeable work-related obstacles Though balancing family and work responsibilities was most commonly selected by both males and females as the first foreseeable obstacle, a greater percentage of female respondents $(1219,55.9 \%$ versus $709,42.6 \%)$ selected this obstacle. In contrast, a greater percentage of male respondents (202, $12.2 \%$ versus $128,5.9 \%)$ identified lack of opportunity/research funding as the top foreseeable obstacle $(p<0.0001)$ (Table 3).

Foreseeable non-work-related responsibilities More male than female respondents expected to be a caretaker to others $(595,33.2 \%$ versus $657,28.2 \%, p=0.0007)$ and financially support others $(1017,56.7 \%$ versus 1184 , $50.9 \%, p=0.0002$ ), respectively (Table 3 ).

\section{Perceptions \\ Intended research/clinical work ratio}

Female students preferred to have no research component $(558,24.3 \%$ versus $348,19.8 \%)$ or $25 \%$-time commitment (1047, 44.6\% versus $747,42.4 \%$ ), while male students preferred 50\% research commitment (309, $17.6 \%$ versus $370,16.1 \%), 75 \%$ research commitment $(319,18.1 \%$ versus $291,12.7 \%)$ or full-time research (38, $2.2 \%$ versus $29,1.3 \%)(p=0.03)$ (Table 4$)$.
Feasibility of research in acute care and surgical specialties More female than male respondents $(750,33.9 \%$ versus $494,29.0 \%$ ) believe that research intensive careers in acute care specialties are feasible, while more male than female respondents $(359,21.1 \%$ versus $359,16.2 \%)$ believe that research intensive careers in acute care are highly difficult $(p<0.0001)$. As for surgical specialties, more females than males perceive research intensive careers as highly feasible (156, 7.1\% versus 98, 5.7\%) or feasible $(707,32.0 \%$ versus $466,27.3 \%)$, while more males than females believe research intensive careers in surgical specialties are highly difficult $(471,27.6 \%$ versus $494,22.3 \%$ ), or impossible $(83,4.9 \%$ versus $56,2.5 \%)$ $(p<0.0001)$ (Table 4$)$.

\section{Perceived important factors in job recruitment}

More female than male respondents perceived connections/networking to be "a great deal of importance" (721, 33.0\% versus 527, 31.5\%), whereas more males than females perceived connections/networking to be of "moderate amount of importance" (406, 24.3\% versus $456,20.9 \%)(p=0.01)$ (Table 4).

\section{Discussion}

There are significant gender disparities in many factors surrounding medical student interest in research. Notably, more males were found to pay for medical school through $\mathrm{MD} / \mathrm{PhD}$ or $\mathrm{DO} / \mathrm{PhD}$ program funding, scholarships, grants, and national service, thus leading to a significantly reduced financial/loan burden. The study also found that more females identify loan repayment as a top foreseeable obstacle to pursuing medical research compared to males, consistent with current literature which states that female matriculation rates consistently remain below 50\% among all MSTP programs [17]. Our data further support this as male respondents were more likely to be enrolled in $\mathrm{MD} / \mathrm{PhD}$ programs ( $25.6 \%$ vs $16.9 \%)$ compared to female respondents.

Furthermore, fewer females identified under-compensation as a top foreseeable obstacle to pursuing a career in research. However, females in the medical profession continue to experience the wage gap which persists through all sectors of society [18]. There may be many reasons for this discordance, one of which may be

\footnotetext{
(See figure on next page.)
}

Fig. 1 a 1st Sector Choice by Gender ${ }^{a}, P=0.0004$, Cramer's $V=0.10 .{ }^{a}$ Top sector choice for participants separated by gender. The following sectors were included in the category "Other" for better visualization: nonprofit, government, industry, and consulting. b 1 st Career Intention by Gender ${ }^{\mathbf{b}}, P<0.0001$, Cramer's V $=0.16{ }^{\mathbf{b}}$ Top career intention for participants separated by gender. The category "Other/NA" was excluded for better visualization. $\mathbf{c} 1$ st Specialty of Interest by Gender ${ }^{c}, P<0.0001$, Cramer's $V=0.31$. ${ }^{\mathbf{c}}$ Top choice specialty of interest for participants separated by gender. The following specialties were included in the category "Medicine" for better visualization: allergy and immunology, dermatology, family medicine, internal medicine, internal medicine subspecialties, medical genetics, pathology, pediatrics, physical medicine and rehabilitation, preventive medicine, and psychiatry. The following specialties were included in the category "Surgery" for better visualization: surgical subspecialties, obstetrics and gynecology, ophthalmology, otolaryngology, and urology. The following specialties were included in the category "Radiology" for better visualization: nuclear medicine and radiation oncology. The category "Other/NA" was excluded for better visualization 
a)

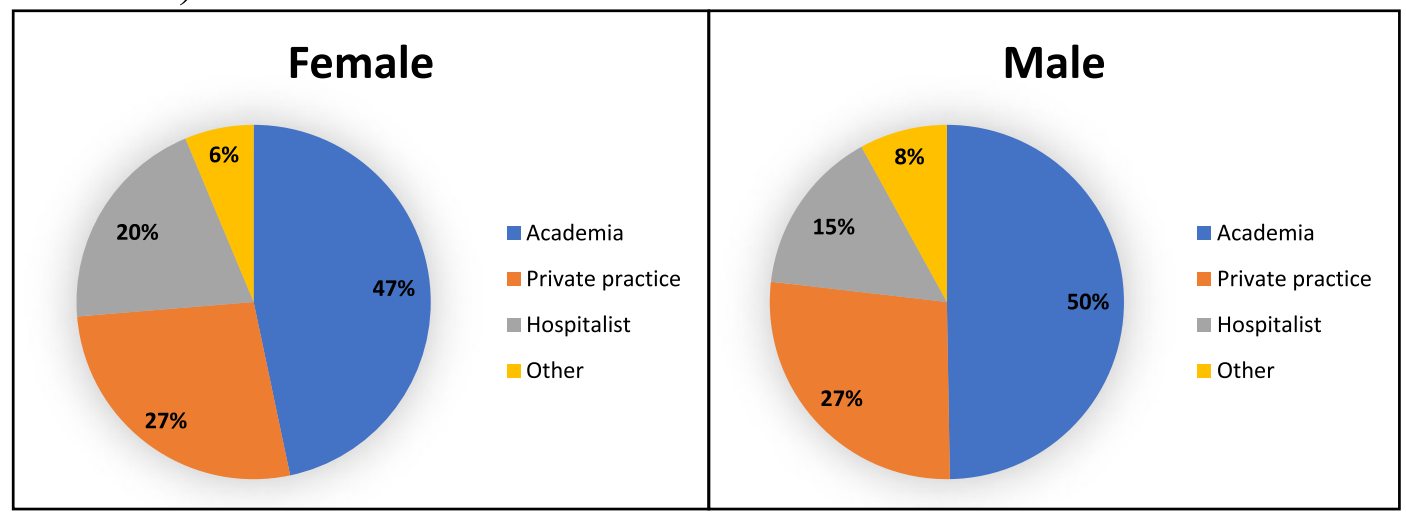

b)

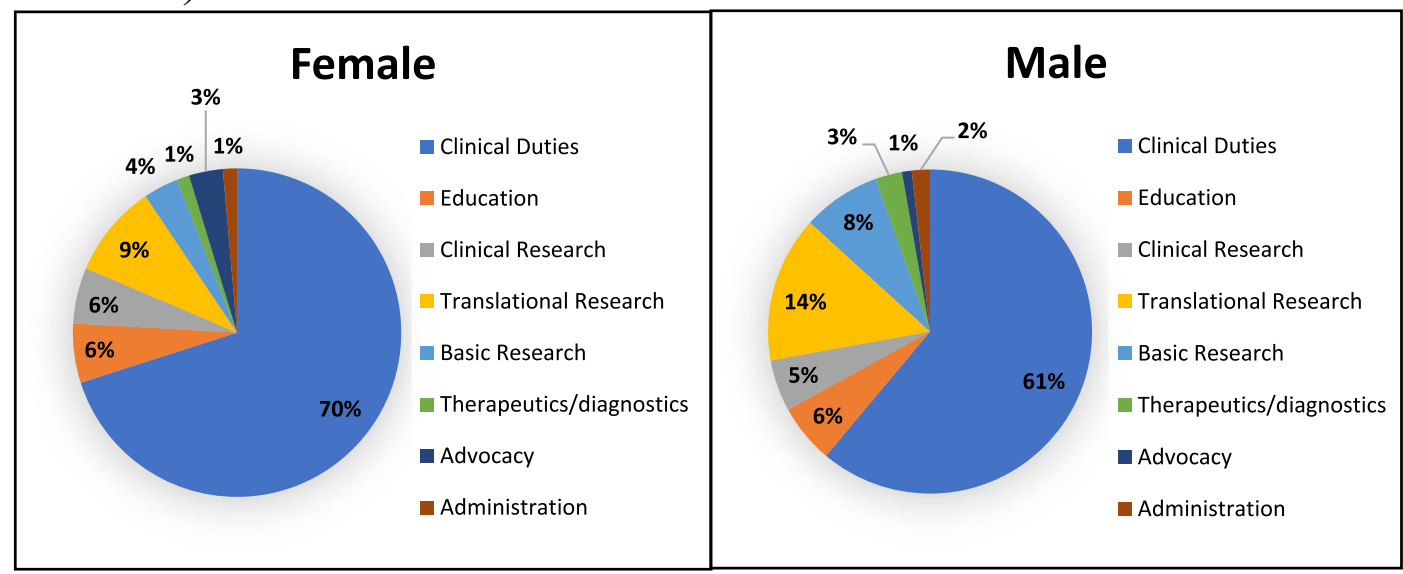

c)

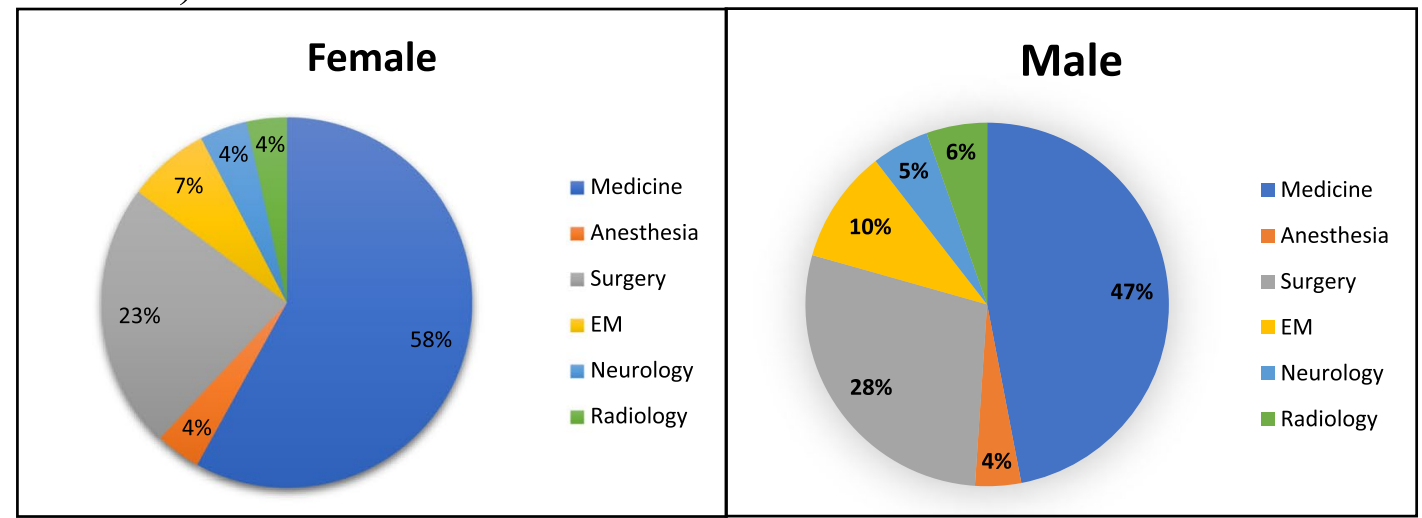

Fig. 1 (See legend on previous page.) 
Table 2 Top Career Selection Factors by Female and Male Respondents

\begin{tabular}{llll}
\hline Factor $^{\mathbf{a}}$ & Female, $\mathbf{n}(\%)$ & Male, $\mathbf{n}(\%)$ & $\mathbf{P}_{\mathbf{0}} \mathbf{0 . 0 0 0 \mathbf { 1 } ^ { \mathbf { b } }}$ \\
\hline Opportunities to do research & $169(7.7 \%)$ & $255(15.4 \%)$ \\
Opportunities for patient care & $809(37.1 \%)$ & $510(30.8 \%)$ \\
Opportunities to teach & $41(1.9 \%)$ & $60(3.6 \%)$ \\
Opportunities for community service & $93(4.3 \%)$ & $29(1.8 \%)$ \\
Opportunities for interaction with students & $20(0.9 \%)$ & $16(1.0 \%)$ \\
Opportunities for travel & $14(0.6 \%)$ & $10(0.6 \%)$ \\
Opportunities for international work & $70(3.2 \%)$ & $42(2.5 \%)$ \\
Opportunities for national work & $8(0.4 \%)$ & $8(0.5 \%)$ \\
Opportunities for local work & $12(0.6 \%)$ & $7(0.4 \%)$ \\
Ability to balance work and personal life & $855(39.2 \%)$ & $539(32.6 \%)$ \\
Financial security & $52(2.4 \%)$ & $110(6.7 \%)$ \\
Autonomy & $33(1.5 \%)$ & $61(3.7 \%)$ \\
Prestige & $7(0.3 \%)$ & $7(0.4 \%)$ \\
TOTAL & $2183(100 \%)$ & $1654(100 \%)$ \\
\hline
\end{tabular}

a Excluding Other/NA

b Male versus female responses were compared using chi-squared tests and Fisher's exact tests where appropriate

c Cramer's $V$ was used to measure effect size between male and female respondents

Table 3 Obstacles by Female and Male Respondents

\begin{tabular}{|c|c|c|c|c|}
\hline Foreseeable work-related responsibilities after residency ${ }^{a}$ & Female, n (\%) & Male, n (\%) & $P<0.0001^{b}$ & Cramer's V $=0.18^{c}$ \\
\hline Lack of opportunity/funding & $128(5.9 \%)$ & $202(12.2 \%)$ & & \\
\hline Not finding position in desired location & $179(8.2 \%)$ & $181(10.9 \%)$ & & \\
\hline Loan repayment & $319(14.6 \%)$ & $210(12.6 \%)$ & & \\
\hline Malpractice/lawsuit & $19(0.9 \%)$ & $42(2.5 \%)$ & & \\
\hline Under-compensation & $65(3.0 \%)$ & $74(4.5 \%)$ & & \\
\hline Discrimination/biases against your gender, ethnicity, sexual orientation & $34(1.6 \%)$ & $12(0.7 \%)$ & & \\
\hline Sexual harassment & $2(0.1 \%)$ & $0(0.0 \%)$ & & \\
\hline Balancing family and work responsibilities & $1219(55.9 \%)$ & $709(42.6 \%)$ & & \\
\hline Balancing clinical, research, and education responsibilities & $162(7.4 \%)$ & $186(11.2 \%)$ & & \\
\hline Satisfactory professional advancement & $54(2.5 \%)$ & $47(2.8 \%)$ & & \\
\hline TOTAL & $2181(100 \%)$ & $1663(100 \%)$ & & \\
\hline Foreseeable non-work-related responsibilities after residency & & & $P$-Value & Cramer's V \\
\hline Raising children & $2048(88.0 \%)$ & 1579 (88.0\%) & $>0.99$ & N/A \\
\hline Taking care of elderly parents & $1513(65.0 \%)$ & $1150(64.1 \%)$ & 0.54 & N/A \\
\hline Being a caretaker to others & $657(28.2 \%)$ & $595(33.2 \%)$ & 0.0007 & 0.05 \\
\hline Financial support of others & $1184(51.0 \%)$ & $1017(56.7 \%)$ & 0.0002 & 0.06 \\
\hline
\end{tabular}

a Excluding Other/NA

${ }^{\mathrm{b}}$ Male versus female responses were compared using chi-squared tests and Fisher's exact tests where appropriate

' Cramer's V was used to measure effect size between male and female respondents

societal pressure on women to fulfill the heteronormative gender role as the primary caregiver for the family and children, though the contribution of this factor is still debated [13]. Our study further supports this possibility in the finding that a greater percentage of females chose "balancing family and work responsibilities" as the number one factor for both their specialty choice and foreseeable obstacle in pursuing research.

Another notable finding in our study was that while more females saw research intensive careers in surgical and acute care specialties as feasible, fewer females indicated an intent to pursue basic and translational research. 
Table 4 Perceptions of Research/Clinical Work Ratio, Feasibility, and Mentoring

\begin{tabular}{|c|c|c|c|c|}
\hline RI Ratio (Research/Clinical Work) ${ }^{\mathrm{a}}$ & Female, $n(\%)$ & Male, $n(\%)$ & $P=0.03^{b}$ & $\begin{array}{l}\text { Cramer's } \\
\mathrm{V}=0.10^{\mathrm{c}}\end{array}$ \\
\hline $0 \%$ & $558(24.3 \%)$ & $348(19.8 \%)$ & & \\
\hline $25 \%$ & $1047(44.6 \%)$ & $747(42.4 \%)$ & & \\
\hline $50 \%$ & $370(16.1 \%)$ & $309(17.6 \%)$ & & \\
\hline $75 \%$ & $291(12.7 \%)$ & $319(18.1 \%)$ & & \\
\hline $100 \%$ & $29(1.3 \%)$ & $38(2.2 \%)$ & & \\
\hline TOTAL & $2295(100 \%)$ & $1761(100 \%)$ & & \\
\hline How feasible is a research intense career in acute care medicine specialties? & & & $P<0.0001$ & 0.08 \\
\hline Highly feasible & $130(5.9 \%)$ & $118(6.9 \%)$ & & \\
\hline Feasible & $750(33.9 \%)$ & $494(29.0 \%)$ & & \\
\hline Difficult & $945(42.7 \%)$ & $700(41.0 \%)$ & & \\
\hline Highly difficult & $359(16.2 \%)$ & $359(21.1 \%)$ & & \\
\hline Impossible & $30(1.4 \%)$ & $33(1.9 \%)$ & & \\
\hline TOTAL & $2214(100 \%)$ & $1704(100 \%)$ & & \\
\hline How feasible is a research intense career in surgical specialties? & & & $P<0.0001$ & 0.10 \\
\hline Highly feasible & $156(7.1 \%)$ & $98(5.7 \%)$ & & \\
\hline Feasible & $707(32.0 \%)$ & $466(27.3 \%)$ & & \\
\hline Difficult & $799(36.1 \%)$ & $588(34.5 \%)$ & & \\
\hline Highly difficult & $494(22.3 \%)$ & $471(27.6 \%)$ & & \\
\hline Impossible & $56(2.5 \%)$ & $83(4.9 \%)$ & & \\
\hline TOTAL & $2212(100 \%)$ & $1706(100 \%)$ & & \\
\hline \multicolumn{3}{|c|}{$\begin{array}{l}\text { How much importance is given to talents/accomplishments when recruiting applicants for jobs and/or positions } \\
\text { in science and medicine? }\end{array}$} & $P=0.30$ & N/A \\
\hline A great deal of importance & $669(30.7 \%)$ & $519(31.1 \%)$ & & \\
\hline A lot of importance & $1070(49.1 \%)$ & $789(47.2 \%)$ & & \\
\hline Moderate amount of importance & $410(18.8 \%)$ & $327(19.6 \%)$ & & \\
\hline Little importance & $28(1.3 \%)$ & $35(2.1 \%)$ & & \\
\hline None at all & $1(0.1 \%)$ & $1(0.1 \%)$ & & \\
\hline TOTAL & $2178(100 \%)$ & $1671(100 \%)$ & & \\
\hline \multicolumn{3}{|c|}{$\begin{array}{l}\text { How much importance is given to connections/networking when recruiting applicants for jobs and/or positions in } \\
\text { science and medicine? }\end{array}$} & $P=0.01$ & 0.06 \\
\hline A great deal of importance & $721(33.0 \%)$ & $527(31.5 \%)$ & & \\
\hline A lot of importance & $946(43.4 \%)$ & $675(40.4 \%)$ & & \\
\hline Moderate amount of importance & $456(20.9 \%)$ & $406(24.3 \%)$ & & \\
\hline Little importance & $59(2.7 \%)$ & $62(3.7 \%)$ & & \\
\hline None at all & $0(0.0 \%)$ & $2(0.1 \%)$ & & \\
\hline TOTAL & $2182(100 \%)$ & $1672(100 \%)$ & & \\
\hline
\end{tabular}

a Excluding Other/NA

${ }^{b}$ Male versus female responses were compared using chi-squared tests and Fisher's exact tests where appropriate

c Cramer's $V$ was used to measure effect size between male and female respondents

This discrepancy is consistent with the continued minority of women entering surgical and acute care specialties, despite recent parity in absolute numbers of students entering the resident workforce [19].

In conclusion, there are a multitude of factors that contribute to the continued disparities in interest in pursuing a career in medical research among medical students. Although several gender differences were observed, the majority of respondents reported a desire to pursue academic medicine, clinical duties, and medical specialties, along with future expectations to raise children and take care of elderly parents. Notable factors that significantly differed by gender included financial burden from student loans, under-compensation, and work/life balance among other factors. Without concerted efforts to bridge this gap between men and women, these disparities will persist. With the current focus on inclusion and diversification in academic 
medicine, these efforts must target minority populations to ensure these changes come to fruition in the coming years $[20,21]$.

\section{Limitations}

Although this is a large secondary cross-sectional cohort analysis of previously published data, with a total of 4433 respondents from a nationally representative cohort of medical schools, there are a few limitations to this study. First, given the nature of self-reported surveys, there is the inherent limitation of being unable to assess more deeply the motivations behind who chose to respond to the survey, along with the answers of the respondents. Second, with a response rate of $27 \%$, there are limitations to the generalizability of the study results. Third, we did not impute incomplete responses or responses labeled "N/A" to better reflect the answers of all respondents, which we recognize as a statistical limitation of the study. Fourth, many steps have been taken to promote gender equity in recent years, and given that the survey data was collected between 2012 and 2014, the factors considered by students may have changed since that time. However, we believe many of the obstacles discussed in this study are likely still relevant and timely. Fifth, there are inherent limitations to the study given that this was a secondary analysis of previously published data using a survey that was designed to assess factors influencing student interest in research careers, rather than specifically looking at gender differences in these various factors. Finally, a follow-up study investigating the ultimate career choice of respondents would be helpful to gain perspective on how to best address gaps in those pursuing careers in medical research.

\section{Abbreviation}

APSA: American Physician Scientists Association.

\section{Supplementary Information}

The online version contains supplementary material available at https://doi. org/10.1186/s12909-021-03004-z.

Additional file 1.

\section{Acknowledgments}

We would like to thank Skip Brass, Claudia Morrissey, and Mary Lou Schmidt for their helpful comments on this study, and the American Physician Scientists Association (APSA) leadership for their support. We would also like to acknowledge Hajwa Kim, MS for statistical support.

\section{Authors' contributions}

AS and DX analyzed and interpreted the survey data, created the figures and tables, and contributed to writing the manuscript. AS, SE, OT, JD, JMK, and DD contributed to creating the survey instrument and writing the manuscript. All authors read and approved the final manuscript.

\section{Funding}

This project received financial support from the University of Illinois at Chicago Center for Clinical and Translational Science (National Center for Advancing Translational Sciences grant UL1TR002003), the American Physician Scientists Association, the Trustees' Council of Penn Women at the University of Pennsylvania, the Burroughs Wellcome Fund, and the National Heart, Lung, and Blood Institute (grant 5T32HL072742-10 to JMK).

This project received financial support from the University of Illinois at Chicago Center for Clinical and Translational Science (National Center for Advancing Translational Sciences grant UL1TR002003), the American Physician Scientists Association, the Trustees' Council of Penn Women at the University of Pennsylvania, the Burroughs Wellcome Fund, and the National Heart, Lung, and Blood Institute (grant 5T32HL072742-10 to JMK).

\section{Availability of data and materials}

The datasets used and/or analyzed during the current study are available from the corresponding authors on reasonable request.

\section{Declarations}

\section{Ethics approval and consent to participate}

IRB of University of Illinois at Chicago and the University of Pennsylvania exempted the need of ethical approval. Informed consent was obtained from all subjects involved in this study. All methods were carried out in accordance with relevant guidelines and regulations.

\section{Consent for publication}

Not applicable.

\section{Competing interests}

The authors declare no competing interests.

\section{Author details}

${ }^{1}$ Harvard Medical School, Boston, USA. ${ }^{2}$ Keck School of Medicine of USC, LOS Angeles, USA. ${ }^{3}$ David Geffen School of Medicine at UCLA, Los Angeles, USA. ${ }^{4}$ Department of Interventional Radiology, Massachusetts General Hospital, 55 Fruit Street, Boston, MA 02114, USA. ${ }^{5}$ Section of Cardiovascular Medicine, Yale School of Medicine, 333 Cedar Street, New Haven, CT 06510, USA.

Received: 19 May 2021 Accepted: 1 November 2021

Published online: 25 November 2021

\section{References}

1. Funston G, Piper RJ, Connell C, Foden P, Young AM, O'Neill P. Medical student perceptions of research and research-orientated careers: an international questionnaire study. Med Teach. 2016;38(10):1041-8.

2. Laurent L, Sosenko T, Zamfirova I, Hartwig C. Exploring equality in leadership roles. Physician Leadership J. 2017;4(3):36-40.

3. Chellaiyan VG, Manoharan A, Jasmine M, Liaquathali F. Medical research: perception and barriers to its practice among medical school students of Chennai. J Educ Health Promot. 2019;8.

4. Moraes DW, Jotz M, Menegazzo WR, Menegazzo MS, Veloso S, Machry $M C$, et al. Interest in research among medical students: challenges for the undergraduate education. Rev Assoc Med Bras. 2016;62(7):652-8.

5. Yamazaki Y, Uka T, Shimizu H, Miyahira A, Sakai T, Marui E. Japanese medical students' interest in basic sciences: a questionnaire survey of a medical school in Japan. Tohoku J Exp Med. 2013;229(2):129-36.

6. Biomedical Research Workforce Working Group Report. Natl institutes heal. 2012. https://biomedicalresearchworkforce.nih.gov/docs/Biome dical_research_wgreport.pdf. Accessed 1 May 2021.

7. McGee R Jr, Saran S, Krulwich TA. Diversity in the biomedical research workforce: developing talent. Mount Sinai J Med. 2012;79(3):397-411.

8. Bismark M, Morris J, Thomas L, Loh E, Phelps G, Dickinson H. Reasons and remedies for under-representation of women in medical leadership roles: a qualitative study from Australia. BMJ Open. 2015;5(11). 
9. Shaikh AT, Farhan SA, Siddiqi R, Fatima K, Siddiqi J, Khosa F. Disparity in leadership in neurosurgical societies: a global breakdown. World Neurosurg. 2019;123:95-102.

10. Boulis AK, Jacobs JA. Feminization of an evolving profession. In: The changing face of medicine: women doctors and the evolution of health care in America. Ithaca: Cornell University Press; 2008. p. 25-8.

11. Bickel J, Wara D, Atkinson BF, Cohen LS, Dunn M, Hostler S, et al. Increasing women's leadership in academic medicine: report of the AAMC project implementation committee. Acad Med. 2002;77(10):1043-61.

12. Guelich JM, Singer BH, Castro MC, Rosenberg LE. A gender gap in the next generation of physician-scientists: medical student interest and participation in research. J Invest Med. 2002;50(6):412-8.

13. Edmunds LD, Ovseiko PV, Shepperd S, Greenhalgh T, Frith P, Roberts NW, et al. Why do women choose or reject careers in academic medicine? A narrative review of empirical evidence. Lancet. 2016;388(10062):2948-58.

14. Kwan JM, Daye D, Schmidt ML, Conlon CM, Kim H, Gaonkar B, et al. Exploring intentions of physician-scientist trainees: factors influencing $\mathrm{MD}$ and $\mathrm{MD} / \mathrm{PhD}$ interest in research careers. BMC Med Educ. 2017; 17(1):1-16.

15. Kwan JM, Toubat O, Harrison AM, Riddle M, Wu B, Kim H, et al. A nationwide assessment of perceptions of research-intense academic careers among predoctoral MD and MD-PhD trainees. J Clin Transl Sci. 2020;4(4):307-16. https://doi.org/10.1017/cts.2020.18.

16. Akoglu H. User's guide to correlation coefficients. Turkish J Emerg Med. 2018;18(3):91-3. https://doi.org/10.1016/j.tjem.2018.08.001.

17. Rowell TR, Redd RA, Neuberg DS, Walensky LD. Mind the gap: expediting gender parity in MD-PhD admissions. JCl insight. 2020;5(4).

18. Asgari MM, Carr PL, Bates CK. Closing the gender wage gap and achieving professional equity in medicine. Jama. 2019;321(17):1665-6.

19. de Costa J, Chen-Xu J, Bentounsi Z, Vervoort D. Women in surgery: challenges and opportunities. IJS Glob Health. 2018;1(1):e02.

20. Fuentes-Afflick E. Promoting inclusion in academic medicine. JAMA Netw Open. 2018;1(4):e181010.

21. Soto-Greene M, Culbreath K, Guzman DE, Sánchez JP, Romero-Leggott V. Diversity and inclusion in the academic medicine workforce: encouraging medical students and residents to consider academic careers. MedEdPORTAL. 2018;14.

\section{Publisher's Note}

Springer Nature remains neutral with regard to jurisdictional claims in published maps and institutional affiliations.
Ready to submit your research? Choose BMC and benefit from:

- fast, convenient online submission

- thorough peer review by experienced researchers in your field

- rapid publication on acceptance

- support for research data, including large and complex data types

- gold Open Access which fosters wider collaboration and increased citations

- maximum visibility for your research: over $100 \mathrm{M}$ website views per year

At BMC, research is always in progress.

Learn more biomedcentral.com/submissions 